\title{
RELATOS DE EXPERIÊNCIAS DE ACADÊMICAS DE FISIOTERAPIA EM INTERVENÇÃO PSICOMOTORA COM ADOLESCENTES E ADULTOS COM NECESSIDADES ESPECIAIS DA APAE DE UMA CIDADE DO LITORAL PARANÁ
}

\author{
REPORTS OF EXPERIENCES OF ACADEMIC PHYSIOTHERAPY IN PSYCHOMOTOR \\ INTERVENTION WITH ADOLESCENTS AND ADULTS WITH SPECIAL NEEDS OF THE APAE OF A \\ CITY OF THE COAST OF PARANÁ
}

Franciele Cristina Ferreira de Souza ${ }^{1}$

Manoela de Paula Ferreira ${ }^{2}$

Karize Rafaela Mesquita Novakoski ${ }^{3}$

Vera Lúcia Israel ${ }^{4}$

\begin{abstract}
Resumo
Desde a graduação em Fisioterapia, é relevante apresentar os acadêmicos à prática profissional. Em pessoas com necessidades especiais, o processo de reconhecimento corporal pode estar prejudicado e por meio de atividades motoras, o fisioterapeuta pode auxiliar o processo de aprendizagem motora. $\mathrm{O}$ objetivo deste estudo foi relatar a percepção de acadêmicos de Fisioterapia com intervenções psicomotoras em pessoas com necessidades especiais. Participaram 14 sujeitos com deficiência cognitiva e/ou motora. Após as intervenções, foi possível observar maior identificação número de contornos no desenho. Conclui-se que intervenções psicomotoras podem contribuir para a melhora da percepção corporal e na possível melhora funcional da pessoa com deficiência e que esta atividade foi sensível para apresentar uma fatia do campo profissional aos acadêmicos de Fisioterapia.
\end{abstract}

Palavras-chave: Atividade motora; Imagem corporal; Deficiência.

\begin{abstract}
Since graduation in Physiotherapy, it is relevant to present the students to professional practice. In people with special needs, the body recognition process may be impaired and through motor activities, the physiotherapist can assist the motor learning process. The objective of this study was to report the perception of Physical Therapy academics with psychomotor interventions in people with special needs. Participants were 14 subjects with cognitive and/or motor deficiency. After the interventions, it was possible to observe greater identification number of contours in the drawing. It was concluded that psychomotor interventions can contribute to the improvement of the corporal perception and the possible functional improvement of the disabled person and that this activity was sensitive to present a slice of the professional field to the Physiotherapy students.
\end{abstract}

Keywords: Motor activity; Body Image; Disability.

\footnotetext{
Dossiê: Recebido em 27/09/2017 - Aprovado em 02/01/2018

${ }^{1}$ Fisioterapeuta. $e$-mail: fransouza83@hotmail.com

${ }^{2}$ Fisioterapeuta, doutoranda do Programa de Pós-Graduação em Educação Física da Universidade Federal do Paraná (PGEDF/UFPR).e-mail: manoeladpferreira@gmail.com

${ }^{3}$ Fisioterapeuta, mestrando do PGEDF/UFPR.e-mail: karize.novakoski@gmail.com

4 Fisioterapeuta, Doutora. Professora do Curso de Graduação em Fisioterapia e do PGEDF/UFPR. e-mail: veral.israel@gmail.com (autor correspondente)
} 


\section{Introdução}

O processo de formação profissional deve ser iniciado na Universidade, como forma de assegurar ao acadêmico, noções de autonomia, independência e segurança perante a vida profissional (MOTTER et al., 2014). A Fisioterapia por sua vez atua desde a prevenção até a reabilitação em saúde, passando por setores já consagrados como clínicas e hospitais, até escolas, comunidades, empresas, lares de longa permanência. Com os projetos de extensão, muitas vezes os acadêmicos passam a ter contato com a comunidade e nela desempenhar seu papel como auxiliador do processo de organização social e contribuição com o ambiente. $\mathrm{O}$ curso de Fisioterapia da UFPR apresenta essa proposta integradora, ensino, pesquisa e extensão, como forma de contribuir para a formação humana e integral dos acadêmicos (SIGNORELLI et al., 2010).

Sabendo disso, dentro da Universidade foram criados vários projetos para atender diversas demandas da sociedade, com foco na melhora coletiva para facilitar a diversidade e inclusão. Existem vários centros para pessoas com deficiência no Litoral do Paraná que fizeram parcerias com a Universidade. Por sua vez, os professores e acadêmicos criaram diversos projetos, dentre eles, este de extensão com foco no estimulo motor para pessoas com deficiência múltipla que, muitas vezes tem limitação na funcionalidade motora e no reconhecimento do próprio corpo, fato que prejudica as atividades de vida diária,

A auto percepção da imagem corporal de um indivíduo ocorre de maneira contínua desde a infância até a idade adulta (MACHADO; CARVALHO; MALUCELLI, 2014). Existem diversos fatores que influenciam neste processo como por exemplo fatores físicos, psicológicos, ambientais, comportamentais. Assim podemos dizer que as experiências vividas pelo indivíduo poderão aprimorar sua percepção corporal ao longo da vida (CORREIA; FALQUETO; GOMES, 2014; FROES et al., 2010). Tanto a percepção corporal quanto a percepção do corpo no tempo e no espaço, fazem parte dos fatores que colaboram para o desenvolvimento de aspectos motores, físicos e cognitivos do ser humano (ROSA NETO; AMARO; PRESTES, 2011).
Indivíduos com necessidades especiais poderão sofrer um processo desarmônico de desenvolvimento emocional, social, cognitivo e escolar, devido à uma percepção corporal prejudicada (ROSA NETO; AMARO; PRESTES, 2011). Além disso, indivíduos que apresentam dificuldades na percepção corporal podem apresentar situações de ansiedade, tensão, insegurança o que as fazem posicionar-se de maneira desigual perante ao grupo de indivíduos da mesma idade prejudicando assim a adaptação sócio-afetiva (BORGES; RUBIO, 2013).

Crianças com desenvolvimento motor atípico podem apresentar déficits motores relacionados ao controle, coordenação e reconhecimento do movimento até a idade adulta, portanto faz-se necessária a identificação e o acompanhamento das reais necessidades funcionais e possíveis alterações na percepção corporal (WILLRICH; AZEVEDO; FERNANDES, 2008). Estes indivíduos podem necessitar de atividades lúdicas, desafiadoras que incitem a realização de ações corporais para facilitar o desenvolvimento da percepção corporal (BERTOLDI; LADEWIG; ISRAEL, 2007). Assim, a psicomotricidade pode ser adotada como uma estratégia para desenvolver a funcionalidade do indivíduo (SANTOS, 2016). Ela existe em todas as atividades que acompanham o desenvolvimento da motricidade do indivíduo permitindo assim o conhecimento e domínio do próprio corpo (BORGES; RUBIO, 2013).

Uma das técnicas utilizadas para analisar a percepção corporal é a avaliação por meio do desenho da figura humana. Esta técnica é comumente utilizada nas avaliações psicológicas e avalia aspectos cognitivos, emocionais e orgânicos do indivíduo (VIAPIANA, 2016). Além de aspectos visuo-táteis, cinestésicos e psico-afetivos (BERTOLDI, 2004). Contudo, esta técnica também pode avaliar como o corpo é percebido pelo indivíduo e a relação do corpo com o mundo que o rodeia (MATOS; COSTA; MACHADO, 2014), pois o desenho pode expressar o repertório conceitual do corpo humano, bem como nos permite avaliar processos mentais como orientação espacial, memória de detalhes, coordenação viso-motora (SUEHIRO; BENFICA; CARDIM, 2016).

Desta forma, este estudo teve como objetivo relatar a percepção de acadêmicos de Fisioterapia ao trabalhar com intervenções psicomotoras em pessoas 
com necessidades especiais frequentadoras da APAE de uma cidade do Litoral do Paraná.

\section{Metodologia}

Esta pesquisa é do tipo intervencional, transversal, qualitativa e quantitativa (HOCKMAN et al. 2005). Foi realizada por meio de um projeto de extensão do curso de Fisioterapia da Universidade Federal do Paraná, intitulado "Intervenção Psicomotora em Crianças e Idosos no Litoral do Paraná", número 101/11E do ano de 2011. As intervenções ocorreram em uma APAE de uma cidade do litoral do Paraná e foram respeitados os preceitos éticos de pesquisa com seres humanos, segundo a resolução 196/96.

Como forma de intervenção, as acadêmicas do curso de Fisioterapia organizaram propostas atividades psicomotoras com os recursos visuais, motores e sensitivos a partir dos recursos da instituição, que permitiram que os participantes tivessem oportunidades de reconhecimento corporal de diversas formas lúdicas. Estas atividades foram organizadas em parceria com fisioterapeutas, pedagogos, educadores físicos, cuidadores e profissionais da dança.

Cada frequentador da APAE realizou uma única intervenção e as avaliações ocorreram antes e imediatamente após a intervenção. A intervenção foi aplicada apenas uma vez em cada sala, a qual é dividida de acordo com a faixa etária dos sujeitos. Participaram da pesquisa 4 turmas heterogêneas da APAE. As intervenções tiveram a duração de 60 minutos. As avaliações continham a aplicação do desenho da figura humana (KOLCH, 1984) e a MIF (MONTEIRO et al.,2013) antes e após intervenção psicomotora, sendo que a MIF foi aplicada apenas na avaliação inicial com caráter de classificação funcional.

O estudo foi aplicado nos meses de Junho a Outubro de 2011 e a metodologia aplicada seguiu conforme apresentado na figura 1 .

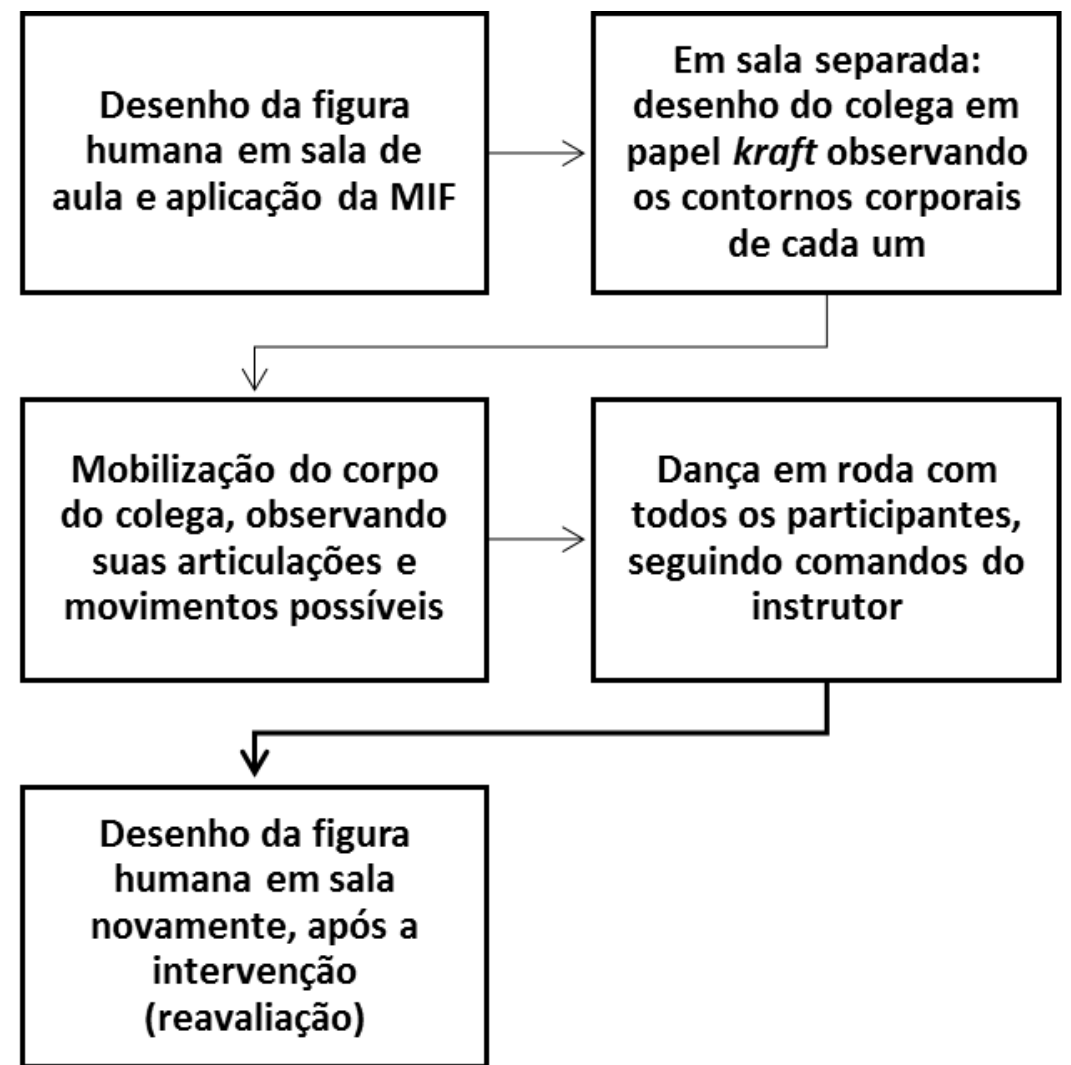

FIGURA 1 - FLUXOGRAMA DO PROGRAMA DE INTERVENÇÃO 
Para a avaliação do desenho da figura humana foi dado o mesmo comando em todas as salas, este era: Desenhe nesta folha você, como você é, as partes de cima: cabeça, braços e mãos; as partes do meio: barriga, costas, peito e as partes de baixo: pernas e pés. Este comando era dado para todos os alunos que o ouviam sentados em suas carteiras com folha e lápis na mão, quando o mesmo terminava seu desenho em seguida uma avaliadora retirava o material. O aluno tinha liberdade de virar a folha da forma que quisesse para realizar o desenho e as cores dos lápis eram dadas de maneira aleatória.

A escala MIF (Medida de Independência Funcional) foi utilizada para melhor caracterizar a independência funcional de cada participante, fazendo com que cada um deles fosse identificado e avaliado considerando sua capacidade funcional, sendo assim a tarefa a ele solicitada estaria de acordo com suas particularidades. Esta escala pontua o desempenho do indivíduo em algumas tarefas demandadas pelo dia a dia. É constituída por 18 tarefas referentes ao autocuidado, controle de esfíncter, transferências, locomoção, comunicação e cognição social (BORBETTA; ASSIS, 2016). Esta é classificada de 1 a 7 pontos, sendo 1 o mais dependente e 7 o mais independente, e seu escore varia de 18 a 126 indicando que quanto mais alto, maior é o grau de independência funcional (MONTEIRO, et al., 2013).

Para esta pesquisa, a aplicação da escala foi realizada por meio de entrevista direta. O entrevistador era uma pesquisadora (acadêmica) devidamente treinada e conhecedora da escala, e o entrevistado era a professora que trabalha diretamente com o sujeito da pesquisa o qual passa 4 $\mathrm{h} /$ dia, 5 dias/semana e o acompanha em todas as tarefas solicitadas e realizadas durante o teste. Esta escala foi aplicada no primeiro dia, antes da intervenção como meio para que os pesquisadores conhecessem a realidade dos alunos dessa instituição de ensino especial e pudessem fazer uma caracterização da amostra.

As atividades das intervenções eram realizadas de forma lúdica, uma vez com cada turma, com materiais confeccionados pelas acadêmicas participantes do projeto, e estas eram acompanhadas pela professora responsável do projeto e pela fisioterapeuta da instituição. As intervenções tinham duração de 60 minutos, cada pesquisadora era responsável por no máximo dois sujeitos.

Para a obtenção das médias estatísticas dos valores atribuídos a cada sujeito com a escala de Medida Funcional (MIF) utilizamos o software Excel. Já para análise dos desenhos da figura humana utilizamos a metodologia qualitativa com descrição das imagens e comparação entre o pré e pós-intervenção.

\section{Resultados e discussão}

Participaram da pesquisa 14 sujeitos pré e pós uma intervenção psicomotora com ênfase na percepção corporal, baseados no desenho da figura humana. As idades variam entre 11 e 51 anos, 8 eram do gênero masculino e 6 do feminino, com deficiências físico-motoras e cognitivas variadas, dentre elas: Síndrome de Down (S. Down), Encefalopatia Crônica não Progressiva da Infância (ECNPI), mais conhecida como Paralisia Cerebral (PC), Esquizofrenia, Autismo e alteração cognitiva não identificada.

A análise da escala MIF foi utilizada como base para caracterização da amostra obtendo média funcional de 104,2 pontos entre os 14 participantes do projeto sendo que o menor valor obtido foi de 66 e o maior foi de 126 (Tabela 1), maior resultado obtido pela escala de acordo com a graduação descrita por Talmelli et al. (2010). Tais resultados demostram que todos os participantes tinham maior independência para suas atividades funcionais, porém em sua maioria dependem de terceiros para supervisionar funções do dia-a-dia.

A análise dos resultados do desenho da figura humana foi feita de forma qualitativa a partir da percepção das alunas e da professora, fisioterapeuta doutora em educação especial. Para a análise dos desenhos considerou-se o maior número de contornos, o aumento de suas formas e a diferenciação de segmentos corporais. Além destes aspectos foi observado na análise pré e pós intervenção 4 níveis de graduação do desenho: 1) não realiza o desenho ou realiza um desenho desintegrado, sem organização gráfica e praticamente irreconhecível; 2) realiza um desenho muito pequeno ou muito grande, desproporcional e com pobreza significativa de partes anatômicas; 3 ) 
realiza um desenho completo, simétrico, com alterações pequenas faciais e com extremidades com leves distorções, e 4) realiza um desenho perfeito em proporção, rico anatomicamente e com disposição espacial correta (FONSECA, 1995, citado por BERTOLDI, 2004).

TABELA 1 - CARACTERIZAÇÃO DOS SUJEITOS DA PESQUISA

\begin{tabular}{ccclc}
\hline SUJEITO & GÊNERO & IDADE & \multicolumn{1}{c}{ PATOLOGIA } & MIF \\
\hline 1 & Feminino & 37 & Esquizofrenia e epilepsia & 82 \\
2 & Feminino & 30 & CID. F.70.1 G.40.2 (Epilepsia com crises complexas) & 109 \\
3 & Masculino & 50 & Paralisia cerebral não especificada & 122 \\
4 & Masculino & 54 & Autismo & 122 \\
5 & Feminino & 47 & Sem diagnostico fechado & 82 \\
6 & Masculino & 51 & Epilepsia de difícil controle e déficit cognitivo & 99 \\
7 & Masculino & 24 & Autismo & 66 \\
8 & Masculino & 14 & Síndrome de Down & 108 \\
9 & Masculino & 12 & Síndrome de Down & 126 \\
10 & Masculino & 11 & Sem diagnóstico fechado & 115 \\
11 & Feminino & 12 & Síndrome de Down & 113 \\
12 & Feminino & 14 & Sem diagnóstico fechado & 110 \\
13 & Feminino & 15 & Síndrome de Down & 109 \\
14 & Masculino & 12 & Disfasia cerebral de expressão em consequência de alterações & 97
\end{tabular}

Fonte: As autoras (2017)

De maneira geral em todos os desenhos foi observado um maior número de contornos e partes do corpo representadas após a intervenção psicomotora em comparação com os desenhos préintervenção. Tais alterações que podem representar o maior reconhecimento corporal e atenção para segmentos antes negligenciados. A seguir são apresentados alguns dos desenhos pré e pós intervenção realizados pelos 14 participantes.

No desenho da Figura 2 é possível perceber o maior número de formas, com melhor aproveitamento do espaço da folha, porém a imagem ainda não apresenta organização gráfica do corpo humano.

Na Figura 3 podemos observar da mesma forma que na Figura 2 um aumento no número de contornos e tamanhos, porém não é mantido uma grafia coerente com a figura humana após uma intervenção psicomotora.

A Figura 4 nos mostra um maior aproveitamento do papel, maiores contornos, sugerindo um esboço da cabeça humana e tronco, porém ainda carente de maiores contornos corporais.

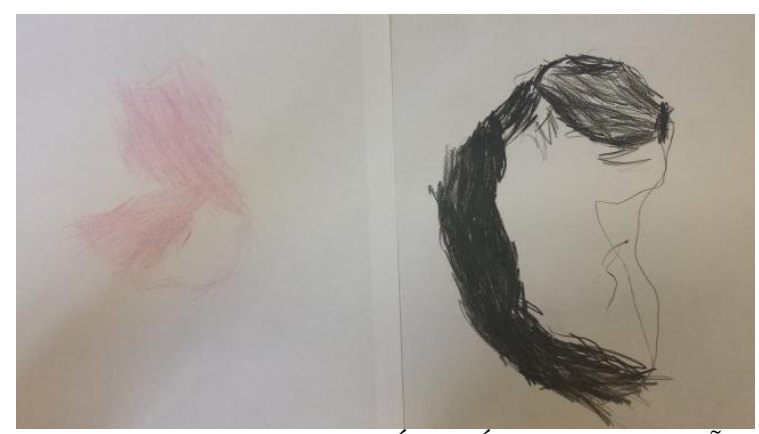

FIGURA 2 - DESENHO PRÉ E PÓS INTERVENÇÃO DO PARTICIPANTE 11 COM 12 ANOS E SÍNDROME DE DOWN

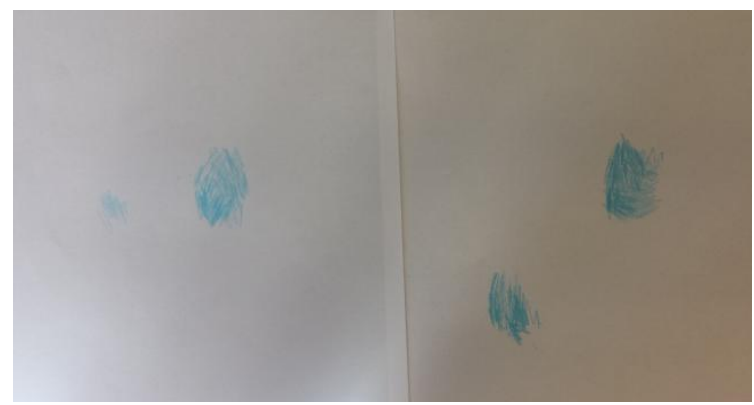

FIGURA 3 - DESENHO PRÉ E PÓS INTERVENÇÃO DO PARTICIPANTE 8 COM 14 ANOS E SÍNDROME DE DOWN 


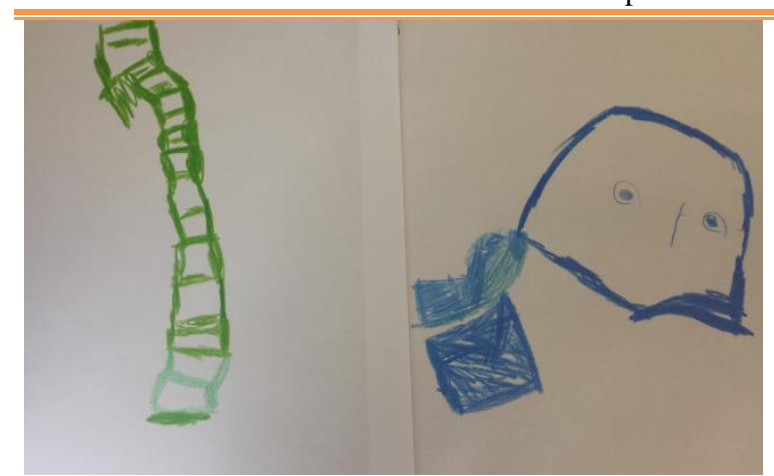

FIGURA 4 - DESENHO PRÉ E PÓS INTERVENÇÃO DO PARTICIPANTE 9, COM 9 ANOS E SÍNDROME DE DOWN

As acadêmicas participantes desta pesquisa conseguiram compreender a relevância dos trabalhos desenvolvidos em diversos níveis de atenção à saúde. Observaram que mesmo em presença de uma deficiência, é possível aos poucos mudar perspectivas e aprimorar habilidades. Além disso, viram sua futura profissão em ação em um contexto escolar e se envolveram com profissionais da educação e da saúde.

Com o aumento da expectativa de vida, pessoas com deficiência também estão vivendo mais e a sociedade cada vez mais busca estar preparada para incluir a diversidade humana (SILVEIRA et al., 2011). Nesta perspectiva, estratégias psicomotoras no ambiente escolar, visando a funcionalidade motora e independência são estratégias para o envolvimento da pessoa com deficiência na sociedade.

Durante a análise dos desenhos observaram-se alguns com omissões de partes do corpo. Em seu estudo Fukamachi et al. (2010), apresentam a identificação da imagem corporal em duas adolescentes modelos e evidenciam omissão de algumas partes do corpo e apontam esta falha como uma demonstração de dificuldade quanto ao próprio esquema corporal. Esta situação corrobora com os achados em nosso estudo onde muitos dos sujeitos apresentam omissões em seu desenho, este fato pode estar ligado a não vivencia de algumas atividades motoras, pois como apresentado por Fróis e Moreira (2010) a imagem corporal é adquirida durante toda a vida e está relacionada com as experiências corporais e psicossociais vividas, e talvez por nosso público ser de pessoas com algum tipo de deficiência intelectual associado ao motor, este fato pode ser justificado.
Dessa forma a intervenção com atividades psicomotoras é necessária para melhorias no repertório motor de pessoas com deficiência, podendo proporcionar uma melhora na percepção corporal e consequentemente aprimorando o seu conhecimento do corpo e de sua imagem corporal seguindo um ciclo de conhecimento, vivencia e aprendizado, o que pode contribuir com as atividades do dia a dia (SAMPAIO et al., 2013).

O fato de observarmos o aumento nos contornos nos desenhos pós intervenção psicomotora pode estar associado ao aumento do repertório motor dos sujeitos a partir da experimentação de novos movimentos e vivências, além do relacionamento inter e intrapessoal durante o estudo, dessa forma reforçamos a idéia de uma melhor atenção a atividades psicomotoras para pessoas com algum deficiência.

Mesmo sabendo que a personalidade está parcialmente formada aos cinco anos (CARDOSO; CAPITÃO, 2009) e com ela o reconhecimento na noção da percepção corporal, a experiência psicomotora desta pesquisa pode ter proporcionado uma nova versão de muitas representações corporais. Provavelmente pela percepção por meio da visão, audição, tato, olfato do corpo humano.

Neste sentido de aprendizagem pelo movimento, podemos ver que mesmo muitos dos sujeitos avaliados neste estudo não tendo a linguagem oral bem desenvolvida, o que pode segundo Cardoso e Capitão (2009) interferir no processo de reconhecimento de si e do seu corpo no espaço, o movimento proposto na intervenção resultou em uma nova significação comprovada pelo desenho da figura humana.

Fonseca, Vecchi e Gama (2012) em seu trabalho utilizaram a dança como ferramenta para a abordagem da identificação da imagem corporal. Como eles em nosso estudo utilizamos a dança em uma das vertentes da psicomotricidade para tal objetivo, buscando por meio dela apresentar novas experiências aos indivíduos visto que a musicalidade pode auxiliar no processo de percepção do corpo no espaço trazido por movimentos ainda não utilizados.

A percepção corporal é resultado das relações neurológicas comportamentais e sensoriais na relação com o ambiente e é construída pela experiência do movimento do corpo em diferentes 
condições (BERTOLDI; LADEWIG; ISRAEL, 2007). Sabendo disso, na pessoa com deficiência, devemos buscar ferramentas de intervenção que busquem por meio de estratégias de direcionamento de atenção, o reconhecimento corporal de forma lúdica e objetiva, para facilitar o processo de reconhecimento corporal.

O desenho da figura humana se mostrou um bom instrumento para observação da percepção corporal de pessoas com deficiência freqüentadores da APAE, por representar a "consciência do corpo" resultado das aprendizagens psicomotoras dadas ao longo da vida (BERTOLDI, 2004).

\section{Conclusão}

Com este trabalho conclui-se que as intervenções psicomotoras realizadas de forma coletiva, respeitando a especificidade de cada sujeito, podem ser benéficas para a possível melhora da percepção corporal.

O trabalho com a psicomotricidade também se mostrou eficaz com a maior participação dos sujeitos durante a intervenção, pois estes aderiram cada vez mais às atividades ao longo da proposta de intervenção e com os pesquisadores quando estes voltavam à APAE. Porém sugere-se que tais trabalhos sejam desenvolvidos de forma contínua, para que os participantes possam realmente explorar todo o potencial individual de aprendizagem.

Vale ressaltar que tais achados foram observados em pessoas com deficiência múltipla, demonstrando assim que atividades motoras adequadas a faixa etária são capazes de promover mudança na atitude de reconhecimento corporal. Tal estratégia aponta para a necessidade da experiência corporal para todos os tipos de deficiência e a possível melhora funcional advinda da socialização e vivência corporal.

\section{Referências}

BERTOLDI, A. L. S. A influência do uso de dicas de aprendizagem na percepção corporal de crianças portadoras de deficiência motora. 2004. 137 f. Dissertação (Mestrado em Educação Física) Departamento de Educação Física, Universidade Federal do Paraná, Curitiba, 2004.
BERTOLDI, A. L. S; LADEWIG, I; ISRAEL, V. L. Influência da seletividade de atenção no desenvolvimento da percepção corporal de crianças com deficiência motora. Rev. Bras. Fisioter., São Carlos, v. 1, n. 4, p. 319-324, 2007.

BORBETTA, D. C.; ASSIS, M. R. Reprodutibilidade, validade e responsividade da escala de Medida de Independência Funcional (MIF) na lesão medular: revisão de literatura. Acta Fisiatr., v. 15, n. 3, p. 176-181, 2008.

BORGES, M. F.; RUBIO, J. A. S. A educação psicomotora como instrumento no processo de aprendizagem. Revista Eletrônica Saberes da Educação, v. 4, n. 1, 2013.

CARDOSO, L. M.; CAPITÃO, C. G. Evidências de validade do Teste do Desenho da Figura Humana para o contexto da surdez. Aval. psicol., Porto Alegre, v. 8, n. 2, p. 245-254, ago. 2009.

CORREIA, J. S. C.; FALQUETO, P. S.; GOMES, S. C. A. Percepção corporal de adolescentes de uma escola pública de Porto Velho/RO. Ensaios e Ciência: ciências biológicas, agrárias e da saúde, v. 17 , n. 1 , p. $79-88,2014$

FONSECA, C. C.; VECCHI, R. L.; GAMA, E. F. A influencia da dança de salão na percepção corporal, Rev. Motriz, Rio Claro, v. 18, p. 200-2007, jan./mar. 2012.

FRÓIS, É. S.; MOREIRA, J. O. A imagem corporal na criança e as novidades do brincar pela internet: um ensaio teórico. Rev. Pesquisas e Práticas Psicossociais, São João del-Rei, v. 5, n. 2, p. 238247, ago./dez. 2010.

FUKAMACHI, K. H.; SILVA A. A.; SILVA, V. X; RAMON, F. M.; SCHWARTZ, L. Percepção da Autoimagem Corporal de Adolescentes Modelos: Dois Estudos de Caso. Psicólogo Informação, v. 14, n. 14, p.80-101, 31 dez. 2010.

HOCHMAN, B.; NAHAS, F. X.; FILHO, R. S. O.; FERREIRA, L. M. Desenhos de pesquisa. Acta Cirurgica Brasileira, v. 20, n. 22, p.2-9, 2005.

KOLCH, O. L. V. Testes projetivos gráficos no diagnóstico psicológico. São Paulo: EPU, 1984.

MACHADO, D. Z.; CARVALHO, D. R.; MALUCELLI, A. Desenvolvimento da imagem corporal interna caracterizado a partir do processo KDD: um estudo sobre órgãos e sistemas. J. Health Inform., v. 6, n. 4, p. 147-52, 2014.

MATOS, S; COSTA, B; MACHADO, C. T. M. O corpo e a imagem corporal em adolescentes: perspectivas a partir do cuidado integral à saúde. Adolesc. Saúde, v. 11, n. 2, p. 19-24, abr./jun. 2014. 
MONTEIRO, R. B. C.; LAURENTINO, G. E. C.; MELO, P. G.; CABRAL, D. L., CORREA, J. C. F.; TEIXEIRA-SALMELA, L. F. Medo de cair e sua relação com a medida da independência funcional $\mathrm{e}$ a qualidade de vida em indivíduos após Acidente Vascular Encefálico. Ciência \& Saúde Coletiva, v. 18, n. 7, p.2017-2027, jul. 2013.

MOTTER, A. A.; VIEIRA, L. A.; BERTOLA, I. P.; FERREIRA, M. P. Sentimentos vivenciados por acadêmicos de Fisioterapia ao estagiar em unidade de terapia intensiva. Cad Edu Saude e Fis., v. 1, n. 2, p. 73-84, 2014.

ROSA NETO, F.; AMARO, K. N.; PRESTES, D. B.; ARAB, C. O esquema corporal de crianças com dificuldade de aprendizagem. Rev. Semestral da Associação Brasileira de Psicologia Escolar e Educacional, v. 15, n. 1, p. 15-22, jan./jun. 2011.

SAMPAIO, L. G.; FRMAKLIN, D. V.; FREIRE, K. L. M.; PEDROSO, N. S. Perfil motor de crianças com Síndrome de Down entre 08 e 11 anos de idade na APAE de Santarém/PA. Fed. Nac. das APAES FENAPES, v. 1, n.2, p. 37-54, 2013.

SANTOS, A. C. A. Psicomotricidade método dirigido e método espontâneo da educação préescolar. 2016. X f. 99 Dissertação (Mestrado em Jogo e Motricidade na Infância) - Escola Superior de Educação de Coimbra/Portugal, 2016.

SIGNORELLI, M. C.; ISRAEL, V. L.; CORRÊA, C. L.; MOTTER, A. A.; TAKEDA, S. Y. M.;
GOMES, A. R. S. Um projeto político-pedagógico de graduação em Fisioterapia pautado em três eixos curriculares. Fisioter Mov., v. 23, n. 2, p. 331-340, 2010.

SILVEIRA, L.; MACAGNAN, J. B. A.; FUCK, J. A. B.; LAGANA, M. T. C. Medida de independência funcional: um desafio para a enfermagem. Rev. Saúde Públ., Florianópolis, v. 4, n. 1, p. 70-83, 2011.

SUEHIRO, A. C. B.; BENFICA, T. S.; CARDIM, N. A. Produção Científica sobre o Teste Desenho da Figura Humana entre 2002 e 2012. Psicol. cienc. prof., v. 36, n. 2, p. 439-448, jun. 2016.

TALMELLI, L. F. S.; GRATÃO, A. C. M.; KUSUMOTA, L.; RODRIGUES, R. A. P.; Nível de independência funcional e déficit cognitivo em idosos com doença de Alzheimer, Rev.Esc. Enferm USP, v. 44, n. 4, p. 233-39, 2010.

VIAPIANA, V. F. Bem-estar subjetivo infantil: avaliação por meio do Desenho da Figura Humana. Avaliação Psicológica, v. 15, n. 1, p. 49-59, 2016.

WILLRICH, A.; AZEVEDO, C. C. F.; FERNANDES, J. O. Desenvolvimento motor na infância: influência dos fatores de risco e programas de intervenção. Rev Neurocienc., p.51-56, 2009. 